\title{
The Matsumoto-Yor Property and Its Converse on Symmetric Cones
}

\author{
Bartosz Kołodziejek ${ }^{1}$
}

Received: 23 June 2015 / Revised: 8 September 2015 / Published online: 7 October 2015

C The Author(s) 2015. This article is published with open access at Springerlink.com

\begin{abstract}
The Matsumoto-Yor (MY) property of the generalized inverse Gaussian and gamma distributions has many generalizations. As was observed in Letac and Wesołowski (Ann Probab 28:1371-1383, 2000), the natural framework for the multivariate MY property is symmetric cones; however, they prove their results for the cone of symmetric positive definite real matrices only. In this paper, we prove the converse to the symmetric cone-variate MY property, which extends some earlier results. The smoothness assumption for the densities of respective variables is reduced to continuity only. This enhancement was possible due to the new solution of a related functional equation for real functions defined on symmetric cones.
\end{abstract}

Keywords Matsumoto-Yor property · Generalized inverse Gaussian distributions . Wishart distributions · Symmetric cones · Hua's identity · Functional equation

Mathematics Subject Classification (2010) Primary 62E10 · Secondary 60E05

\section{Introduction}

Matsumoto and Yor [15,16] have shown that if $X$ and $Y$ are independent random variables, $Y$ is gamma distributed with the shape parameter $p$ and the scale parameter $a$ and $X$ has the generalized inverse Gaussian distribution (GIG) with parameters $(-p, a, b)$, then the random variables $U=(X+Y)^{-1}$ and $V=X^{-1}-(X+Y)^{-1}$ are independent with respective distributions GIG with parameters $(-p, b, a)$ and gamma with parameters $p$ and $b$.

$凶$ Bartosz Kołodziejek

kolodziejekb@mini.pw.edu.pl

1 Faculty of Mathematics and Information Science, Warsaw University of Technology, Pl. Politechniki 1, 00-661 Warsaw, Poland 
Matsumoto and Yor asked about the converse theorem based on the independence of $U$ and $V$. Assume that $X$ and $Y$ are non-degenerate nonnegative independent random variables, such that $U$ and $V$ are independent. Does this imply that $X$ and $Y$ must follow GIG and gamma distributions, respectively?

A positive answer to this question was given by Letac and Wesołowski [13], with the use of Laplace transforms. In the same paper, both the Matsumoto-Yor property and its converse (with additional smoothness assumptions) were generalized to the cone $\Omega_{+}$of symmetric positive definite $(r, r)$ real matrices in the following way. For $p>(r-1) / 2$ and $\mathbf{a}, \mathbf{b} \in \Omega_{+}$, consider two independent random variables $X$ and $Y$ with following densities

$$
\begin{aligned}
\mu_{-p, \mathbf{a}, \mathbf{b}}(\mathrm{d} \mathbf{x}) & =c_{1}(\operatorname{det} \mathbf{x})^{-p-(r+1) / 2} \exp \left(-\operatorname{tr}(\mathbf{a} \cdot \mathbf{x})-\operatorname{tr}\left(\mathbf{b} \cdot \mathbf{x}^{-1}\right)\right) I_{\Omega_{+}}(\mathbf{x}) \mathrm{d} \mathbf{x}, \\
\gamma_{p, \mathbf{a}}(\mathrm{d} \mathbf{y}) & =c_{2}(\operatorname{det} \mathbf{y})^{p-(r+1) / 2} \exp (-\operatorname{tr}(\mathbf{a} \cdot \mathbf{y})) I_{\Omega_{+}}(\mathbf{y}) \mathrm{d} \mathbf{y} .
\end{aligned}
$$

The distribution of $X$ is the GIG with parameters $(-p, \mathbf{a}, \mathbf{b})$, and the distribution of $Y$ is the Wishart distribution with shape parameter $p$ and scale parameter a. Letac and Wesołowski have shown that if $X$ and $Y$ are as above, then $(U, V)$ has distribution $\mu_{-p, \mathbf{b}, \mathbf{a}} \otimes \gamma_{p, \mathbf{b}}$. As was observed by the authors, the natural framework for Matsumoto-Yor property is symmetric cones. Statement of a symmetric cone version of Matsumoto-Yor property is given in Sect. 3.

In this paper, we give a new proof of the converse result of the Matsumoto-Yor property, when $X$ and $Y$ take values in any irreducible symmetric cone. The smoothness assumption is reduced from $C^{2}$ densities in [13] and differentiability in [17] to the continuity only. A new solution of a related functional equation on symmetric cones (see Theorem 4.5) was found under the assumption of continuity of respective functions with the use of the corresponding univariate result due to Wesołowski [18]. Similar reduction in regularity assumptions was recently performed in the density version of Lukacs-Olkin-Rubin in [7].

It is worth mentioning that several related one-dimensional results $[3,10]$ as well as results for random matrices $[9,12]$.

While solving the functional equation, we use Hua's identity, which allows to write the inverse of $V=X^{-1}-(X+Y)^{-1}$ in a very convenient form:

$$
V^{-1}=X+X \cdot Y^{-1} \cdot X
$$

Hua's identity has already proved to be useful in some problems related to GIG and Wishart distributions-see [1], where it was used to analyze some random continued fractions on symmetric cones.

The paper is organized as follows. We start in the next section with some basic definitions and theorems regarding analysis on symmetric cones. In Sect. 3, we define the GIG and Wishart distributions and state the Matsumoto-Yor property on symmetric cones. A core of the proof of the converse to the Matsumoto-Yor property is a solution of some functional equation for real functions with arguments from the cone. Section 4 is devoted to analysis of this functional equation. The statement and the proof of the 
main result are given in Sect. 5. Finally, in Sect. 6, we give some remarks regarding the MY property on matrices of different dimensions and related functional equation.

\section{Symmetric Cones}

In this section, we give a short introduction to the theory of symmetric cones. For further details, we refer to [4].

A Euclidean Jordan algebra is a Euclidean space $\mathbb{E}$ (endowed with the scalar product denoted by $\langle\mathbf{x}, \mathbf{y}\rangle$ ) equipped with a bilinear mapping (product)

$$
\mathbb{E} \times \mathbb{E} \ni(\mathbf{x}, \mathbf{y}) \mapsto \mathbf{x y} \in \mathbb{E}
$$

and a neutral element $\mathbf{e}$ in $\mathbb{E}$ such that for all $\mathbf{x}, \mathbf{y}, \mathbf{z}$ in $\mathbb{E}$ :

- $\mathbf{x y}=\mathbf{y x}$,

- $\mathbf{x}\left(\mathbf{x}^{2} \mathbf{y}\right)=\mathbf{x}^{2}(\mathbf{x y})$

- $x e=x$

- $\langle\mathbf{x}, \mathbf{y z}\rangle=\langle\mathbf{x y}, \mathbf{z}\rangle$.

For $\mathbf{x} \in \mathbb{E}$ let $\mathbb{L}(\mathbf{x}): \mathbb{E} \rightarrow \mathbb{E}$ be the linear map defined by

$$
\mathbb{L}(\mathbf{x}) \mathbf{y}=\mathbf{x y},
$$

and define

$$
\mathbb{P}(\mathbf{x})=2 \mathbb{L}^{2}(\mathbf{x})-\mathbb{L}\left(\mathbf{x}^{2}\right)
$$

Let $\operatorname{End}(\mathbb{E})$ denote the space of endomorphisms of $\mathbb{E}$. The map $\mathbb{P}: \mathbb{E} \mapsto \operatorname{End}(\mathbb{E})$ is called the quadratic representation of $\mathbb{E}$.

An element $\mathbf{x}$ is said to be invertible if there exists an element $\mathbf{y}$ in $\mathbb{E}$ such that $\mathbb{L}(\mathbf{x}) \mathbf{y}=\mathbf{e}$. Then, $\mathbf{y}$ is called the inverse of $\mathbf{x}$ and it is denoted by $\mathbf{y}=\mathbf{x}^{-1}$. Note that the inverse of $\mathbf{x}$ is unique. It can be shown that $\mathbf{x}$ is invertible if and only if $\mathbb{P}(\mathbf{x})$ is invertible, and in this case, $(\mathbb{P}(\mathbf{x}))^{-1}=\mathbb{P}\left(\mathbf{x}^{-1}\right)$.

A Euclidean Jordan algebra $\mathbb{E}$ is said to be simple if it is not a Cartesian product of two Euclidean Jordan algebras of positive dimensions. Up to linear isomorphism, there are only five kinds of Euclidean simple Jordan algebras. Let $\mathbb{K}$ denote either the real numbers $\mathbb{R}$, the complex ones $\mathbb{C}$, the quaternions $\mathbb{H}$ or the octonions $\mathbb{O}$. Let us write $S_{r}(\mathbb{K})$ for the space of $r \times r$ Hermitian matrices valued in $\mathbb{K}$, endowed with the Euclidean structure $\langle\mathbf{x}, \mathbf{y}\rangle=$ Trace $(\mathbf{x} \cdot \overline{\mathbf{y}})$ and with the Jordan product

$$
\mathbf{x y}=\frac{1}{2}(\mathbf{x} \cdot \mathbf{y}+\mathbf{y} \cdot \mathbf{x})
$$

where $\mathbf{x} \cdot \mathbf{y}$ denotes the ordinary product of matrices and $\overline{\mathbf{y}}$ is the conjugate of $\mathbf{y}$. Then $S_{r}(\mathbb{R}), r \geq 1, S_{r}(\mathbb{C}), r \geq 2, S_{r}(\mathbb{H}), r \geq 2$, and the exceptional $S_{3}(\mathbb{O})$ are the first four kinds of Euclidean simple Jordan algebras. Note that in this case if $\mathbb{K} \neq \mathbb{O}$, then

$$
\mathbb{P}(\mathbf{y}) \mathbf{x}=\mathbf{y} \cdot \mathbf{x} \cdot \mathbf{y} .
$$


The fifth kind is the Euclidean space $\mathbb{R}^{n+1}, n \geq 2$, with the Jordan product

$$
\left(x_{0}, x_{1}, \ldots, x_{n}\right)\left(y_{0}, y_{1}, \ldots, y_{n}\right)=\left(\sum_{i=0}^{n} x_{i} y_{i}, x_{0} y_{1}+y_{0} x_{1}, \ldots, x_{0} y_{n}+y_{0} x_{n}\right) .
$$

To each Euclidean simple Jordan algebra, one can attach the set $\bar{\Omega}$ of Jordan squares

$$
\bar{\Omega}=\left\{\mathbf{x} \in \mathbb{E}: \text { there exists } \mathbf{y} \text { in } \mathbb{E} \text { such that } \mathbf{x}=\mathbf{y}^{2}\right\} \text {. }
$$

The interior $\Omega$ is a symmetric cone. Moreover, $\Omega$ is irreducible, i.e., it is not the Cartesian product of two convex cones. One can prove that an open convex cone is symmetric and irreducible if and only if it is the symmetric cone $\Omega$ of some Euclidean simple Jordan algebra. Each simple Jordan algebra corresponds to a symmetric cone; hence, there exists up to linear isomorphism also only five kinds of symmetric cones. The cone corresponding to the Euclidean Jordan algebra $\mathbb{R}^{n+1}$ equipped with Jordan product (2) is called the Lorentz cone.

We will now introduce a very useful decomposition in $\mathbb{E}$, called the spectral decomposition. An element $\mathbf{c} \in \mathbb{E}$ is said to be a primitive idempotent if $\mathbf{c c}=\mathbf{c} \neq 0$ and if $\mathbf{c}$ is not a sum of two non-null idempotents. A complete system of primitive orthogonal idempotents is a set $\left(\mathbf{c}_{1}, \ldots, \mathbf{c}_{r}\right)$ such that

$$
\sum_{i=1}^{r} \mathbf{c}_{i}=\mathbf{e} \text { and } \quad \mathbf{c}_{i} \mathbf{c}_{j}=\delta_{i j} \mathbf{c}_{i} \text { for } 1 \leq i \leq j \leq r
$$

The size $r$ of such system is a constant called the rank of $\mathbb{E}$. Any element $\mathbf{x}$ of a Euclidean simple Jordan algebra can be written as $\mathbf{x}=\sum_{i=1}^{r} \lambda_{i} \mathbf{c}_{i}$ for some complete system of primitive orthogonal idempotents $\left(\mathbf{c}_{1}, \ldots, \mathbf{c}_{r}\right)$. The real numbers $\lambda_{i}, i=$ $1, \ldots, r$ are the eigenvalues of $\mathbf{x}$. One can then define the trace and the determinant of $\mathbf{x}$ by, respectively, $\operatorname{tr} \mathbf{x}=\sum_{i=1}^{r} \lambda_{i}$ and det $\mathbf{x}=\prod_{i=1}^{r} \lambda_{i}$. An element $\mathbf{x} \in \mathbb{E}$ belongs to $\Omega$ if and only if all its eigenvalues are strictly positive.

Note that up to a multiplicative constant, $\operatorname{tr}(\mathbf{x y})$ is the only scalar product on $\mathbb{E}$ which makes $\Omega$ self dual. Henceforth, we assume that $\Omega$ is an irreducible cone and that corresponding Jordan algebra $\mathbb{E}$ is equipped with canonical scalar product $\langle\mathbf{x}, \mathbf{y}\rangle=$ $\operatorname{tr}(\mathbf{x y})$.

The rank $r$ and $\operatorname{dim} \Omega$ of irreducible symmetric cone are connected through the relation

$$
\operatorname{dim} \Omega=r+\frac{\mathrm{d} r(r-1)}{2},
$$

where $d$ is an integer called the Peirce constant.

The important property of the determinant is that

$$
\operatorname{det}(\mathbb{P}(\mathbf{x}) \mathbf{y})=(\operatorname{det} \mathbf{x})^{2} \operatorname{det} \mathbf{y}, \quad(\mathbf{x}, \mathbf{y}) \in \Omega^{2} .
$$


It turns out that (3) characterizes determinant-see Lemma 4.2 below. Moreover (see [4, Proposition II.4.2])

$$
\operatorname{Det}(\mathbb{P}(\mathbf{x}))=(\operatorname{det} \mathbf{x})^{2 \operatorname{dim} \Omega / r}
$$

where Det denotes the determinant in the space of endomorphisms on $\Omega$.

In the proof of our main theorem, we will need the following identity (called Hua's identity—see [4, Exercise 5c, p.39])

$$
\mathbf{a}^{-1}-(\mathbf{a}+\mathbf{b})^{-1}=\left(\mathbf{a}+\mathbb{P}(\mathbf{a}) \mathbf{b}^{-1}\right)^{-1}
$$

when $\mathbf{a} \in \Omega, \mathbf{b} \in \mathbb{E}$ are such that $\mathbf{b}, \mathbf{a}+\mathbf{b}$ and $\mathbf{a}+\mathbb{P}(\mathbf{a}) \mathbf{b}^{-1}$ are invertible. Note that if $\mathbf{a}, \mathbf{b} \in \Omega$, then $\mathbf{a}^{-1}-(\mathbf{a}+\mathbf{b})^{-1} \in \Omega$. For the cone $\Omega_{+}$of symmetric positive definite real matrices, Hua's identity takes the form given in (1).

\section{Wishart and GIG Distributions}

The Wishart distribution $\gamma_{p, \mathbf{a}}$ in $\bar{\Omega}$ is defined for any $\mathbf{a} \in \Omega$ and any $p$ in the set

$$
\Lambda=\{0, d / 2, d, \ldots, d(r-1) / 2\} \cup(d(r-1) / 2, \infty)
$$

by its Laplace transform

$$
\int_{\bar{\Omega}} \exp (-\langle\sigma, \mathbf{y}\rangle) \gamma_{p, \mathbf{a}}(\mathrm{d} \mathbf{y})=\left(\frac{\operatorname{det} \mathbf{a}}{\operatorname{det}(\mathbf{a}+\sigma)}\right)^{p},
$$

which holds for any $\sigma+\mathbf{a} \in \Omega$. If $p>\operatorname{dim} \Omega / r-1$, then $\gamma_{p}, \mathbf{a}$ is absolutely continuous with respect to the Lebesgue measure and has the density

$$
\gamma_{p, \mathbf{a}}(\mathrm{d} \mathbf{x})=\frac{(\operatorname{det} \mathbf{a})^{p}}{\Gamma_{\Omega}(p)}(\operatorname{det} \mathbf{x})^{p-\operatorname{dim} \Omega / r} \mathrm{e}^{-\langle\mathbf{a}, \mathbf{x}\rangle} I_{\Omega}(\mathbf{x}) \mathrm{d} \mathbf{x}, \quad \mathbf{x} \in \Omega,
$$

where $\Gamma_{\Omega}$ is the gamma function of the symmetric cone $\Omega$ (see [4, p.124]).

The absolutely continuous generalized inverse Gaussian distribution $\mu_{p, \mathbf{a}, \mathbf{b}}$ on $\Omega$ is defined for $\mathbf{a}, \mathbf{b} \in \Omega$ and $p \in \mathbb{R}$ by its density

$$
\mu_{p, a, b}(\mathrm{~d} \mathbf{x})=\frac{1}{K_{p}(\mathbf{a}, \mathbf{b})}(\operatorname{det} \mathbf{x})^{p-\operatorname{dim} \Omega / r} \mathrm{e}^{-\langle\mathbf{a}, \mathbf{x}\rangle-\left\langle\mathbf{b}, \mathbf{x}^{-1}\right\rangle} I_{\Omega}(\mathbf{x}) \mathrm{d} \mathbf{x}, \quad \mathbf{x} \in \Omega,
$$

where $K_{p}(\mathbf{a}, \mathbf{b})$ is a normalizing constant.

In [13], Theorem 3.1 was proved in the special case of the cone of symmetric positive definite real matrices $\Omega_{+}$. As it was observed by the authors, symmetric cones are the natural framework for considering the Matsumoto-Yor property. We state the following theorem without a proof as it only mimics the argument for $\Omega_{+}$. The original proof relies on the properties of Bessel-like functions $\left(K_{p}(\mathbf{a}, \mathbf{b})\right)$ introduced in [5], which retain their usual properties in the symmetric cone setting. 
Theorem 3.1 Let $p \in \Lambda$ and $\boldsymbol{a}$ and $\boldsymbol{b}$ in irreducible symmetric cone $\Omega$. Let $X$ and $Y$ be independent random variables in $\Omega$ and $\bar{\Omega}$ with respective distributions $\mu_{-p, \boldsymbol{a}, \boldsymbol{b}}$ and $\gamma_{p, a}$. Then random variables $U=(X+Y)^{-1}$ and $V=X^{-1}-(X+Y)^{-1}$ are independent with respective distributions $\mu_{-p, \boldsymbol{b}, \boldsymbol{a}}$ and $\gamma_{p, \boldsymbol{b}}$.

\section{Functional Equations}

At the beginning of this section, we state three results that will be useful in the proof of the main technical result-Theorem 4.5. The first one regards regular additive functions (see [11]) on symmetric cone.

Lemma 4.1 (Additive Cauchy functional equation) Let $f: \Omega \rightarrow \mathbb{R}$ be a measurable function such that

$$
f(\boldsymbol{x})+f(\boldsymbol{y})=f(\boldsymbol{x}+\boldsymbol{y}), \quad(\boldsymbol{x}, \boldsymbol{y}) \in \Omega^{2} .
$$

Then there exists $\boldsymbol{f} \in \mathbb{E}$ such that $f(\boldsymbol{x})=\langle\boldsymbol{f}, \boldsymbol{x}\rangle$ for any $\boldsymbol{x} \in \Omega$.

An elementary proof of this theorem may be found in [6]. The following lemma was recently proved in [8].

Lemma 4.2 (Logarithmic Pexider functional equation) Let $f_{1}, f_{2}, f_{3}: \Omega \rightarrow \mathbb{R}$ be measurable functions such that

$$
f_{1}(\boldsymbol{x})+f_{2}(\boldsymbol{y})=f_{3}\left(\mathbb{P}\left(\boldsymbol{x}^{1 / 2}\right) \boldsymbol{y}\right), \quad(\boldsymbol{x}, \boldsymbol{y}) \in \Omega^{2} .
$$

Then there exist a constant $q \in \mathbb{R}$ and constants $\gamma_{1}, \gamma_{2} \in \mathbb{R}$ such that for all $\boldsymbol{x} \in \Omega$,

$$
\begin{aligned}
& f_{1}(\boldsymbol{x})=q \log \operatorname{det} \boldsymbol{x}+\gamma_{1}, \\
& f_{2}(\boldsymbol{x})=q \log \operatorname{det} \boldsymbol{x}+\gamma_{2}, \\
& f_{3}(\boldsymbol{x})=q \log \operatorname{det} \boldsymbol{x}+\gamma_{1}+\gamma_{2} .
\end{aligned}
$$

The main technical result will rely on the following univariate result due to Wesołowski [18].

Theorem 4.3 Let $A, B, C$ and $D$ be locally integrable real functions defined on $(0, \infty)$ such that

$$
g(x(x+y))-g(y(x+y))=\alpha(x)-\alpha(y), \quad(x, y) \in(0, \infty)^{2} .
$$

Then there exist real numbers $A, B, C$ and $D$ such that for any $x>0$,

$$
g(x)=A x+B \log x+C, \quad \alpha(x)=A x^{2}+B \log x+D
$$

The following result then follows from Theorem 4.3. 
Theorem 4.4 Let $A, B, C$ and $D$ be locally integrable real functions defined on $(0, \infty)$ such that

$$
A(x)+B(y)=C\left((x+y)^{-1}\right)+D\left(x^{-1}-(x+y)^{-1}\right), \quad(x, y) \in(0, \infty)^{2} .
$$

Then there exist real numbers $p, f, g$ and $C_{i}, i=1, \ldots, 4$, such that for any $x>0$,

$$
\begin{aligned}
& A(x)=-p \log x+f x+g x^{-1}+C_{1}, \\
& B(x)=p \log x+f x+C_{2}, \\
& C(x)=-p \log x+g x+f x^{-1}+C_{3}, \\
& D(x)=p \log x+g x+C_{4},
\end{aligned}
$$

and $C_{1}+C_{2}=C_{3}+C_{4}$.

Proof Denote $g_{1}(x)=A\left(x^{-1}\right)-B\left(x^{-1}\right)$ and $\alpha_{1}(x)=D\left(x^{2}\right)$. Interchange the roles of $x$ and $y$ in (7) and subtract from the original equation. Then

$$
g_{1}\left(x^{-1}\right)-g_{1}\left(y^{-1}\right)=\alpha_{1}\left(\sqrt{\frac{y}{x(x+y)}}\right)-\alpha_{1}\left(\sqrt{\frac{x}{y(x+y)}}\right) .
$$

Inserting $x=(u(u+v))^{-1}$ and $y=(v(u+v))^{-1}$, we arrive at (6) with $g$ and $\alpha$ replaced, respectively, with $g_{1}$ and $\alpha_{1}$.

Substituting $x \mapsto(x+y)^{-1}$ and $y \mapsto x^{-1}-(x+y)^{-1}$ in (7), we obtain

$$
A\left((x+y)^{-1}\right)+B\left(x^{-1}-(x+y)^{-1}\right)=C(x)+D(y), \quad(x, y) \in(0, \infty)^{2} .
$$

As before, denoting $g_{2}(x)=C\left(x^{-1}\right)-D\left(x^{-1}\right)$ and $\alpha_{2}(x)=B\left(x^{2}\right)$ and subtracting the same equation with $x$ and $y$ interchanged, we see that (6) holds true for $g_{2}$ and $\alpha_{2}$ also. Functions $g_{i}$ and $\alpha_{i}, i=1,2$, are locally integrable, because for $g_{1}$ we have

$$
\int_{K}\left|A\left(x^{-1}\right)-B\left(x^{-1}\right)\right| \mathrm{d} x=\int_{\phi(K)}|A(y)-B(y)| \frac{\mathrm{d} y}{y^{2}} \leq c \int_{\phi(K)}|A(y)-B(y)| \mathrm{d} y
$$

for all compact sets $K \subset(0, \infty)$, where $\phi(K)$ is the (compact) image of $K$ under $\phi(x)=x^{-1}$. Since $A$ and $B$ were assumed to be locally integrable, we see that $g_{1}$ is locally integrable. Analogously, we proceed for $g_{2}, \alpha_{1}$ and $\alpha_{2}$. Thus, by Theorem 4.3, we obtain (we borrow this notation from Theorem 4.3):

$$
\begin{aligned}
B(x) & =\alpha_{2}(\sqrt{x})=A_{2} x+B_{2} / 2 \log x+D_{2}, \\
D(x) & =\alpha_{1}(\sqrt{x})=A_{1} x+B_{1} / 2 \log x+D_{1}, \\
A(x) & =A(x)-B(x)+B(x)=g_{1}\left(x^{-1}\right)+\alpha_{2}(\sqrt{x}) \\
& =A_{2} x+A_{1} x^{-1}-\left(B_{1}-B_{2} / 2\right) \log x+C_{1}+D_{2},
\end{aligned}
$$




$$
\begin{aligned}
C(x) & =C(x)-D(x)+D(x)=g_{2}\left(x^{-1}\right)+\alpha_{1}(\sqrt{x}) \\
& =A_{1} x+A_{2} x^{-1}-\left(B_{2}-B_{1} / 2\right) \log x+C_{2}+D_{1},
\end{aligned}
$$

Inserting it back into (7), it can be quickly verified that $B_{1}=B_{2}=B$.

We are now ready to state and solve the functional equation related to the MatsumotoYor property on symmetric cones.

Theorem 4.5 Let $a, b, c$ and $d$ be continuous real functions defined on $\Omega$ such that

$$
a(\boldsymbol{x})+b(\boldsymbol{y})=c\left((\boldsymbol{x}+\boldsymbol{y})^{-1}\right)+d\left(\boldsymbol{x}^{-1}-(\boldsymbol{x}+\boldsymbol{y})^{-1}\right), \quad(\boldsymbol{x}, \boldsymbol{y}) \in \Omega^{2} .
$$

Then there exist constants $q \in \mathbb{R}, \boldsymbol{f}, \boldsymbol{g} \in \mathbb{E}$ and $\gamma_{i} \in \mathbb{R}, i=1,2,3$, such that for any $x \in \Omega$,

$$
\begin{aligned}
& a(\boldsymbol{x})=q \log \operatorname{det} \boldsymbol{x}+\langle\boldsymbol{f}, \boldsymbol{x}\rangle+\left\langle\boldsymbol{g}, \boldsymbol{x}^{-1}\right\rangle+\gamma_{1}+\gamma_{3}, \\
& b(\boldsymbol{x})=-q \log \operatorname{det} \boldsymbol{x}+\langle\boldsymbol{f}, \boldsymbol{x}\rangle+\gamma_{2}, \\
& c(\boldsymbol{x})=q \log \operatorname{det} \boldsymbol{x}+\langle\boldsymbol{g}, \boldsymbol{x}\rangle+\left\langle\boldsymbol{f}, \boldsymbol{x}^{-1}\right\rangle+\gamma_{3}, \\
& d(\boldsymbol{x})=-q \log \operatorname{det} \boldsymbol{x}+\langle\boldsymbol{g}, \boldsymbol{x}\rangle+\gamma_{1}+\gamma_{2} .
\end{aligned}
$$

Proof By inserting $(\mathbf{x}, \mathbf{y})=(\alpha \mathbf{z}, \beta \mathbf{z})$ for $\alpha, \beta>0$ and $\mathbf{z} \in \Omega$ into (8), we arrive at the equation (7) with $A(\alpha):=a(\alpha \mathbf{z}), B(\alpha):=b(\alpha \mathbf{z}), C(\alpha):=c\left(\alpha \mathbf{z}^{-1}\right)$ and $D(\alpha):=$ $d\left(\alpha \mathbf{z}^{-1}\right)$. Functions $A, B, C$ and $D$ are continuous, so they are locally integrable. Therefore, by Theorem 4.4, for any $\mathbf{z} \in \Omega$, there exist constants $p(\mathbf{z}), f(\mathbf{z}), g(\mathbf{z})$ and $C_{i}(\mathbf{z}), i=1, \ldots, 4$, such that

$$
\begin{aligned}
a(\alpha \mathbf{z}) & =-p(\mathbf{z}) \log \alpha+f(\mathbf{z}) \alpha+g(\mathbf{z}) \alpha^{-1}+C_{1}(\mathbf{z}), \\
b(\alpha \mathbf{z}) & =p(\mathbf{z}) \log \alpha+f(\mathbf{z}) \alpha+C_{2}(\mathbf{z}), \\
c\left(\alpha \mathbf{z}^{-1}\right) & =-p(\mathbf{z}) \log \alpha+g(\mathbf{z}) \alpha+f(\mathbf{z}) \alpha^{-1}+C_{3}(\mathbf{z}), \\
d\left(\alpha \mathbf{z}^{-1}\right) & =p(\mathbf{z}) \log \alpha+g(\mathbf{z}) \alpha+C_{4}(\mathbf{z}), \\
C_{1}(\mathbf{z})+C_{2}(\mathbf{z}) & =C_{3}(\mathbf{z})+C_{4}(\mathbf{z}),
\end{aligned}
$$

for any $\alpha>0$ and $\mathbf{z} \in \Omega$. Functions $\mathbf{z} \mapsto p(\mathbf{z}), \mathbf{z} \mapsto f(\mathbf{z}), \mathbf{z} \mapsto g(\mathbf{z})$ and $\mathbf{z} \mapsto$ $C_{i}(\mathbf{z}), i=1, \ldots, 4$, are continuous, because $a, b, c$ and $d$ are continuous. Let $\beta>0$. By the equality $a(\alpha(\beta \mathbf{z}))=a((\alpha \beta) \mathbf{z})$, we obtain that for any $\alpha>0$,

$$
\begin{aligned}
a(\alpha \beta \mathbf{z}) & =-p(\mathbf{z}) \log \alpha \beta+f(\mathbf{z}) \alpha \beta+g(\mathbf{z}) \alpha^{-1} \beta^{-1}+C_{1}(\mathbf{z}) \\
& =-p(\beta \mathbf{z}) \log \alpha+f(\beta \mathbf{z}) \alpha+g(\beta \mathbf{z}) \alpha^{-1}+C_{1}(\beta \mathbf{z}),
\end{aligned}
$$

hence

$$
\begin{aligned}
& f(\beta \mathbf{z})=\beta f(\mathbf{z}), \quad g(\beta \mathbf{z})=\beta^{-1} g(\mathbf{z}), \\
& p(\beta \mathbf{z})=p(\mathbf{z}), \quad C_{1}(\beta \mathbf{z})=C_{1}(\mathbf{z})-p(\mathbf{z}) \log \beta .
\end{aligned}
$$


Following the same procedure for functions $b, c$ and $d$, we have

$$
\begin{aligned}
& C_{i}(\beta \mathbf{z})=C_{i}(\mathbf{z})+p(\mathbf{z}) \log \beta, \quad i=2,3, \\
& C_{4}(\beta \mathbf{z})=C_{4}(\mathbf{z})-p(\mathbf{z}) \log \beta .
\end{aligned}
$$

Using (9) for $\alpha=1$ in (8), we get

$$
\begin{aligned}
f(\mathbf{x})+g(\mathbf{x})+C_{1}(\mathbf{x})+f(\mathbf{y})+C_{2}(\mathbf{y})= & g(\mathbf{x}+\mathbf{y})+f(\mathbf{x}+\mathbf{y})+C_{3}(\mathbf{x}+\mathbf{y}) \\
& +g\left(\left(\mathbf{x}^{-1}-(\mathbf{x}+\mathbf{y})^{-1}\right)^{-1}\right) \\
& +C_{4}\left(\left(\mathbf{x}^{-1}-(\mathbf{x}+\mathbf{y})^{-1}\right)^{-1}\right) .
\end{aligned}
$$

Consider the above equation for $\left(\alpha^{-1} \mathbf{x}, \alpha^{-1} \mathbf{y}\right) \in \Omega^{2}, \alpha>0$. Then, by (10),

$$
\begin{aligned}
& \alpha^{-1} f(\mathbf{x})+\alpha g(\mathbf{x})+C_{1}\left(\alpha^{-1} \mathbf{x}\right)+\alpha^{-1} f(\mathbf{y})+C_{2}\left(\alpha^{-1} \mathbf{y}\right) \\
& =\alpha g(\mathbf{x}+\mathbf{y})+\alpha^{-1} f(\mathbf{x}+\mathbf{y})+C_{3}\left(\alpha^{-1}(\mathbf{x}+\mathbf{y})\right) \\
& \quad+\alpha g\left(\left(\mathbf{x}^{-1}-(\mathbf{x}+\mathbf{y})^{-1}\right)^{-1}\right)+C_{4}\left(\alpha^{-1}\left(\mathbf{x}^{-1}-(\mathbf{x}+\mathbf{y})^{-1}\right)^{-1}\right) .
\end{aligned}
$$

Multiplying both sides of the above equation by $\alpha$ and passing to the limit as $\alpha \rightarrow 0$, by (11), we obtain

$$
\begin{aligned}
& f(\mathbf{x})+f(\mathbf{y})-f(\mathbf{x}+\mathbf{y}) \\
&=\lim _{\alpha \rightarrow 0} \alpha\left\{C_{3}\left(\alpha^{-1}(\mathbf{x}+\mathbf{y})\right)+C_{4}\left(\alpha^{-1}\left(\mathbf{x}^{-1}-(\mathbf{x}+\mathbf{y})^{-1}\right)^{-1}\right)\right. \\
&\left.-C_{1}\left(\alpha^{-1} \mathbf{x}\right)-C_{2}\left(\alpha^{-1} \mathbf{y}\right)\right\} .
\end{aligned}
$$

By (10) and (11), the limit on the right-hand side of the above equation equals 0 . Thus, by Lemma 4.1, there exists $\mathbf{f} \in \mathbb{E}$ such that $f(\mathbf{x})=\langle\mathbf{f}, \mathbf{x}\rangle$. Analogously, consider (12) for $(\alpha \mathbf{x}, \alpha \mathbf{y}) \in \Omega^{2}, \alpha>0$, multiply its both sides by $\alpha$ and pass to the limit as $\alpha \rightarrow 0$. Then

$$
\begin{gathered}
g(\mathbf{x})-g(\mathbf{x}+\mathbf{y})-g\left(\left(\mathbf{x}^{-1}-(\mathbf{x}+\mathbf{y})^{-1}\right)^{-1}\right) \\
=\lim _{\alpha \rightarrow 0} \alpha\left\{C_{3}(\alpha(\mathbf{x}+\mathbf{y}))+C_{4}\left(\alpha\left(\mathbf{x}^{-1}-(\mathbf{x}+\mathbf{y})^{-1}\right)^{-1}\right)\right. \\
\left.\quad-C_{1}(\alpha \mathbf{x})-C_{2}(\alpha \mathbf{y})\right\}=0 .
\end{gathered}
$$

Define $\bar{g}(\mathbf{x})=g\left(\mathbf{x}^{-1}\right)$. Then,

$$
\bar{g}\left(\mathbf{x}^{-1}\right)=\bar{g}\left((\mathbf{x}+\mathbf{y})^{-1}\right)+\bar{g}\left(\mathbf{x}^{-1}-(\mathbf{x}+\mathbf{y})^{-1}\right) .
$$

Thus, $\bar{g}$ is additive, i.e., there exists $\mathbf{g} \in \mathbb{E}$ such that $g(\mathbf{x})=\left\langle\mathbf{g}, \mathbf{x}^{-1}\right\rangle$. 
By the use of above results for $f$ and $g$, (12) simplifies to

$$
C_{1}(\mathbf{x})+C_{2}(\mathbf{y})=C_{3}(\mathbf{x}+\mathbf{y})+C_{4}\left(\left(\mathbf{x}^{-1}-(\mathbf{x}+\mathbf{y})^{-1}\right)^{-1}\right) .
$$

Recall that by Hua's identity (5), the argument of $C_{4}$ above may be written as

$$
\left(\mathbf{x}^{-1}-(\mathbf{x}+\mathbf{y})^{-1}\right)^{-1}=\mathbf{x}+\mathbb{P}(\mathbf{x}) \mathbf{y}^{-1}
$$

Using this fact along with (11) in (13) for $\mathbf{y}=\alpha \mathbf{z}$, we obtain

$$
\begin{aligned}
& C_{1}(\mathbf{x})+C_{2}(\mathbf{z})+p(\mathbf{z}) \log \alpha \\
& \quad=C_{1}(\mathbf{x})+C_{2}(\alpha \mathbf{z})=C_{3}(\mathbf{x}+\alpha \mathbf{z})+C_{4}\left(\alpha^{-1}\left(\alpha \mathbf{x}+\mathbb{P}(\mathbf{x}) \mathbf{z}^{-1}\right)\right) \\
& =C_{3}(\mathbf{x}+\alpha \mathbf{z})+C_{4}\left(\alpha \mathbf{x}+\mathbb{P}(\mathbf{x}) \mathbf{z}^{-1}\right)+p\left(\alpha \mathbf{x}+\mathbb{P}(\mathbf{x}) \mathbf{z}^{-1}\right) \log \alpha .
\end{aligned}
$$

Passing to the limit as $\alpha \rightarrow 0$ (recall that $C_{i}$ are continuous on $\Omega$ ), we obtain

$$
C_{1}(\mathbf{x})+C_{2}(\mathbf{z})-C_{3}(\mathbf{x})-C_{4}\left(\mathbb{P}(\mathbf{x}) \mathbf{z}^{-1}\right)=\lim _{\alpha \rightarrow 0} \log \alpha\left\{p\left(\alpha \mathbf{x}+\mathbb{P}(\mathbf{x}) \mathbf{z}^{-1}\right)-p(\mathbf{z})\right\}
$$

for any $(\mathbf{x}, \mathbf{z}) \in \Omega^{2}$. A necessary condition for the limit on the right-hand side to exist is

$$
\lim _{\alpha \rightarrow 0}\left\{p\left(\alpha \mathbf{x}+\mathbb{P}(\mathbf{x}) \mathbf{z}^{-1}\right)-p(\mathbf{z})\right\}=0
$$

But $p$ is continuous and $\lim _{\alpha \rightarrow 0} p\left(\alpha \mathbf{x}+\mathbb{P}(\mathbf{x}) \mathbf{z}^{-1}\right)=p\left(\mathbb{P}(\mathbf{x}) \mathbf{z}^{-1}\right)$, hence $p(\mathbf{z})=$ $p\left(\mathbb{P}(\mathbf{x}) \mathbf{z}^{-1}\right)$. Thus, function $p$ is constant and the right-hand side of (14) is equal to 0 . Hence, substituting $\mathbf{z}=\mathbf{y}^{-1}$ and $\mathbf{x} \mapsto \mathbf{x}^{1 / 2}$ in (14), we get

$$
C_{1}\left(\mathbf{x}^{1 / 2}\right)-C_{3}\left(\mathbf{x}^{1 / 2}\right)+C_{2}\left(\mathbf{y}^{-1}\right)=C_{4}\left(\mathbb{P}\left(\mathbf{x}^{1 / 2}\right) \mathbf{y}\right) .
$$

Define $f_{1}(\mathbf{x}):=C_{1}\left(\mathbf{x}^{1 / 2}\right)-C_{3}\left(\mathbf{x}^{1 / 2}\right), f_{2}(\mathbf{x}):=C_{2}\left(\mathbf{x}^{-1}\right)$ and $f_{3}(\mathbf{x}):=C_{4}(\mathbf{x})$ for $\mathbf{x} \in \Omega$. Then

$$
f_{1}(\mathbf{x})+f_{2}(\mathbf{y})=f_{3}\left(\mathbb{P}\left(\mathbf{x}^{1 / 2}\right) \mathbf{y}\right), \quad(\mathbf{x}, \mathbf{y}) \in \Omega^{2} .
$$

By Lemma 4.2, there exist real constants $q, \gamma_{1}$ and $\gamma_{2}$ such that for any $\mathbf{x} \in \Omega$,

$$
\begin{aligned}
& f_{1}(\mathbf{x})=q \log \operatorname{det} \mathbf{x}+\gamma_{1}, \\
& f_{2}(\mathbf{x})=q \log \operatorname{det} \mathbf{x}+\gamma_{2}, \\
& f_{3}(\mathbf{x})=q \log \operatorname{det} \mathbf{x}+\gamma_{1}+\gamma_{2},
\end{aligned}
$$


that is,

$$
\begin{aligned}
& C_{1}(\mathbf{x})=C_{3}(\mathbf{x})+2 q \log \operatorname{det} \mathbf{x}+\gamma_{1}, \\
& C_{2}(\mathbf{x})=-q \log \operatorname{det} \mathbf{x}+\gamma_{2}, \\
& C_{4}(\mathbf{x})=q \log \operatorname{det} \mathbf{x}+\gamma_{1}+\gamma_{2} .
\end{aligned}
$$

Let us go back to (13) and use the above result. Then

$$
\begin{aligned}
& C_{3}(\mathbf{x})+2 q \log \operatorname{det} \mathbf{x}-q \log \operatorname{det} \mathbf{y} \\
& \quad=C_{3}(\mathbf{x}+\mathbf{y})+q \log \operatorname{det}\left(\mathbf{x}+\mathbb{P}(\mathbf{x}) \mathbf{y}^{-1}\right), \quad(\mathbf{x}, \mathbf{y}) \in \Omega^{2} .
\end{aligned}
$$

Since $\operatorname{det}\left(\mathbf{x}+\mathbb{P}(\mathbf{x}) \mathbf{y}^{-1}\right)=\operatorname{det}\left(\mathbf{x}^{2}\right) \operatorname{det}\left(\mathbf{x}^{-1}+\mathbf{y}^{-1}\right)$, we obtain

$$
C_{3}(\mathbf{x})-q \log \operatorname{det} \mathbf{y}=C_{3}(\mathbf{x}+\mathbf{y})+q \log \operatorname{det}\left(\mathbf{x}^{-1}+\mathbf{y}^{-1}\right) .
$$

One can interchange $\mathbf{x}$ and $\mathbf{y}$ on the right-hand side to obtain

$$
C_{3}(\mathbf{x})+q \log \operatorname{det} \mathbf{x}=C_{3}(\mathbf{y})+q \log \operatorname{det} \mathbf{y}=\text { const }:=\gamma_{3},
$$

that is, $C_{3}(\mathbf{x})=-q \log \operatorname{det} \mathbf{x}+\gamma_{3}$, what completes the proof.

\section{Main Result}

In the following section, we prove our main result, which is a converse to the Matsumoto-Yor property in the symmetric cone-variate case. We reduce the smoothness conditions for densities from $C^{2}$ densities in [13] and differentiability in [17] to the continuity only.

Theorem 5.1 Let $X$ and $Y$ be independent random variables in $\Omega$ with continuous and strictly positive densities. If the random variables $U=(X+Y)^{-1}$ and $V=$ $X^{-1}-(X+Y)^{-1}$ are independent, then there exists $p>\operatorname{dim} \Omega / r-1, \boldsymbol{a}$ and $\boldsymbol{b}$ in $\Omega$ such that $X$ and $Y$ follow respective distributions $\mu_{-p, \boldsymbol{a}, \boldsymbol{b}}$ and $\gamma_{p, \boldsymbol{a}}$.

Proof Define the map $\Psi: \Omega^{2} \rightarrow \Omega^{2}$ by $\Psi(\mathbf{x}, \mathbf{y})=\left((\mathbf{x}+\mathbf{y})^{-1}, \mathbf{x}^{-1}-(\mathbf{x}+\mathbf{y})^{-1}\right)=$ $(\mathbf{u}, \mathbf{v})$. Obviously, $(U, V)=\Psi(X, Y)$. Function $\Psi$ is a bijection. In order to find the joint density of $(U, V)$, the essential computation is the one involved with finding the Jacobian $J$ of the map $\psi^{-1}$, that is, the determinant of the linear map

$$
\left(\begin{array}{l}
\mathrm{d} \mathbf{u} \\
\mathrm{d} \mathbf{v}
\end{array}\right) \mapsto\left(\begin{array}{l}
\mathrm{d} \mathbf{x} \\
\mathrm{d} \mathbf{y}
\end{array}\right)=\left(\begin{array}{l}
\mathrm{d} \mathbf{x} / \mathrm{d} \mathbf{u} \mathrm{d} \mathbf{x} / \mathrm{d} \mathbf{v} \\
\mathrm{d} \mathbf{y} / \mathrm{d} \mathbf{u} \mathrm{d} \mathbf{y} / \mathrm{d} \mathbf{v}
\end{array}\right)\left(\begin{array}{l}
\mathrm{d} \mathbf{u} \\
\mathrm{d} \mathbf{v}
\end{array}\right)
$$


It is easy to see that $\Psi=\Psi^{-1}$, that is $(\mathbf{x}, \mathbf{y})=\left((\mathbf{u}+\mathbf{v})^{-1}, \mathbf{u}^{-1}-(\mathbf{u}+\mathbf{v})^{-1}\right)$. Note that the derivative of the map $\mathbf{x} \mapsto \mathbf{x}^{-1}$ is $-\mathbb{P}(\mathbf{x})^{-1}$. Thus

$$
\begin{aligned}
J & =\left|\begin{array}{cc}
-\mathbb{P}(\mathbf{u}+\mathbf{v})^{-1} & -\mathbb{P}(\mathbf{u}+\mathbf{v})^{-1} \\
-\mathbb{P}(\mathbf{u})^{-1}+\mathbb{P}(\mathbf{u}+\mathbf{v})^{-1} & \mathbb{P}(\mathbf{u}+\mathbf{v})^{-1}
\end{array}\right| \\
& =\left|\begin{array}{cc}
-\mathbb{P}(\mathbf{u})^{-1} & 0 \\
-\mathbb{P}(\mathbf{u})^{-1}+\mathbb{P}(\mathbf{u}+\mathbf{v})^{-1} & \mathbb{P}(\mathbf{u}+\mathbf{v})^{-1}
\end{array}\right| \\
& =\operatorname{Det}\left(\mathbb{P}(\mathbf{u}+\mathbf{v})^{-1} \mathbb{P}(\mathbf{u})^{-1}\right) .
\end{aligned}
$$

By (4), we get

$$
J=(\operatorname{det} \mathbf{u} \operatorname{det}(\mathbf{u}+\mathbf{v}))^{-2 \operatorname{dim} \Omega / r} .
$$

Since $(X, Y)$ and $(U, V)$ have independent components, the following identity holds almost everywhere with respect to the Lebesgue measure:

$$
f_{U}(\mathbf{u}) f_{V}(\mathbf{v})=(\operatorname{det} \mathbf{u} \operatorname{det}(\mathbf{u}+\mathbf{v}))^{-2 \operatorname{dim} \Omega / r} f_{X}\left((\mathbf{u}+\mathbf{v})^{-1}\right) f_{Y}\left(\mathbf{u}^{-1}-(\mathbf{u}+\mathbf{v})^{-1}\right),
$$

where $f_{X}, f_{Y}, f_{U}$ and $f_{V}$ denote densities of $X, Y, U$ and $V$, respectively. Since the respective densities are assumed to be continuous, the above equation holds for every $(\mathbf{u}, \mathbf{v}) \in \Omega^{2}$. Taking the logarithms of both sides of the above equation (it is permitted since $f_{X}, f_{Y}>0$ on $\Omega$ ), we get

$$
a(\mathbf{u})+b(\mathbf{v})=c\left((\mathbf{u}+\mathbf{v})^{-1}\right)+d\left(\mathbf{u}^{-1}-(\mathbf{u}+\mathbf{v})^{-1}\right)
$$

where

$$
\begin{aligned}
a(\mathbf{x}) & =\log f_{U}(\mathbf{x})+\frac{2 \operatorname{dim} \Omega}{r} \log \operatorname{det} \mathbf{x}, \\
c(\mathbf{x}) & =\log f_{X}(\mathbf{x})+\frac{2 \operatorname{dim} \Omega}{r} \log \operatorname{det} \mathbf{x}, \\
b & =\log f_{V}, \quad d=\log f_{Y} .
\end{aligned}
$$

By Theorem 4.5, there exist constants $q \in \mathbb{R}, \mathbf{f}, \mathbf{g} \in \mathbb{E}$ and $\gamma_{i} \in \mathbb{R}, i=1,2,3$, such that for any $\mathbf{x} \in \Omega$,

$$
\begin{aligned}
& c(\mathbf{x})=-q \log \operatorname{det} \mathbf{x}+\langle\mathbf{g}, \mathbf{x}\rangle+\left\langle\mathbf{f}, \mathbf{x}^{-1}\right\rangle+\gamma_{3}, \\
& d(\mathbf{x})=q \log \operatorname{det} \mathbf{x}+\langle\mathbf{g}, \mathbf{x}\rangle+\gamma_{1}+\gamma_{2},
\end{aligned}
$$

that is,

$$
\begin{aligned}
& f_{X}(\mathbf{x})=\mathrm{e}^{\gamma_{3}}(\operatorname{det} \mathbf{x})^{-q-2 \operatorname{dim} \Omega / r} \mathrm{e}^{\langle\mathbf{g}, \mathbf{x}\rangle+\left\langle\mathbf{f}, \mathbf{x}^{-1}\right\rangle}, \\
& f_{Y}(\mathbf{x})=\mathrm{e}^{\gamma_{1}+\gamma_{2}}(\operatorname{det} \mathbf{x})^{q} \mathrm{e}^{\langle\mathbf{g}, \mathbf{x}\rangle} .
\end{aligned}
$$


Since $f_{X}$ and $f_{Y}$ are some densities, we have $\mathbf{a}=-\mathbf{g} \in \Omega, \mathbf{b}=-\mathbf{f} \in \Omega$ and $q=p-\operatorname{dim} \Omega / r>-1$. Thus, $X \sim \mu_{-p, \mathbf{a}, \mathbf{b}}$ and $Y \sim \gamma_{p, \mathbf{a}}$.

\section{Comments}

Recall that $S_{r}(\mathbb{K})$ denotes the space of $r \times r$ Hermitian matrices valued in $\mathbb{K}$. Let $\Omega_{r}(\mathbb{K})$ be the symmetric cone of Jordan algebra $\mathbb{E}=S_{r}(\mathbb{K})$, where $\mathbb{K}$ denotes either the real numbers $\mathbb{R}$, the complex ones $\mathbb{C}$ or the quaternions $\mathbb{H}$. We exclude here the non-associative case $\mathbb{K}=\mathbb{O}$.

Let $z$ be a fixed $s \times r$ matrix of full rank valued in $\mathbb{K}$ and define the linear mapping $\mathbb{P}_{s r}: S_{r}(\mathbb{K}) \rightarrow S_{S}(\mathbb{K})$ by

$$
\mathbb{P}_{s r}(z) \mathbf{x}=z \cdot \mathbf{x} \cdot z^{*}
$$

If $r=s$, then $\mathbb{P}_{s r}$ is the ordinary quadratic representation of $\Omega_{s}$. In the rest of the paper, we will drop the subscript and simply write $\mathbb{P}$ (abusing the notation from previous sections).

Now, consider the following transformation $\psi_{z}: \Omega_{r}(\mathbb{K}) \times \Omega_{s}(\mathbb{K}) \rightarrow \Omega_{s}(\mathbb{K}) \times$ $\Omega_{r}(\mathbb{K})$, where

$$
\psi_{z}(\mathbf{x}, \mathbf{y})=\left((\mathbb{P}(z) \mathbf{x}+\mathbf{y})^{-1}, \mathbf{x}^{-1}-\mathbb{P}\left(z^{*}\right)(\mathbb{P}(z) \mathbf{x}+\mathbf{y})^{-1}\right) .
$$

It is natural to ask whether an analogue of Theorem 5.1 holds if we consider independent random variables $X$ and $Y$ valued in $\Omega_{r}(\mathbb{K})$ and $\Omega_{s}(\mathbb{K})$ and define $(U, V)=\psi_{z}(X, Y)$. The answer is affirmative, and it was given in [14, Theorem 4.1]. Following the same steps as in the proof of Theorem 5.1, the problem of characterization of probability measures is reduced to the problem of solving following functional equation

$$
\begin{aligned}
a(\mathbf{u})+b(\mathbf{v})= & c\left(\left(\mathbb{P}\left(z^{*}\right) \mathbf{u}+\mathbf{v}\right)^{-1}\right)+d\left(\mathbf{u}^{-1}-\mathbb{P}(z)\left(\mathbb{P}\left(z^{*}\right) \mathbf{u}+\mathbf{v}\right)^{-1}\right), \\
& (\mathbf{u}, \mathbf{v}) \in \Omega_{s}(\mathbb{K}) \times \Omega_{r}(\mathbb{K})
\end{aligned}
$$

where $a, d: \Omega_{s}(\mathbb{K}) \rightarrow \mathbb{R}$ and $b, c: \Omega_{r}(\mathbb{K}) \rightarrow \mathbb{R}$ are some unknown functions. This functional equation was solved by Massam and Wesołowski [14] for $\mathbb{K}=\mathbb{R}$ under the assumption that the unknown functions are differentiable. It can be shown that through Theorem 4.5, this assumption may be weakened to continuity. Therefore, we obtain the following refinement of [14, Theorem 4.1]:

Theorem 6.1 Let $X$ and $Y$ be independent random variables with values in $\Omega_{r}(\mathbb{K})$ and $\Omega_{s}(\mathbb{K})$, respectively. Assume that $X$ and $Y$ have continuous densities, which are strictly positive. Define $(U, V)=\psi_{z}(X, Y)$.

If $U$ and $V$ are independent, then there exist matrices $(\boldsymbol{a}, \boldsymbol{b}) \in \Omega_{S}(\mathbb{K}) \times \Omega_{r}(\mathbb{K})$ and a constant $p>\operatorname{dim} \Omega_{r}(\mathbb{K}) / r-1$ such that

$$
(X, Y) \sim \mu_{-p, \mathbb{P}\left(z^{*}\right) \boldsymbol{a}, \boldsymbol{b}}^{(r)} \otimes \gamma_{q, \boldsymbol{a}}^{(s)},
$$


where $q=p+\left(\operatorname{dim} \Omega_{s}(\mathbb{K}) / s-\operatorname{dim} \Omega_{r}(\mathbb{K}) / r\right)$.

The superscripts ${ }^{(s)}$ and ${ }^{(r)}$ are used to emphasize the ranks of the cones on which the distributions are considered.

The solution to (16) was also used in the proof of the characterization of Wishart distribution through its block conditional independence structure (see [14, Theorem 5.1]. One of the technical assumptions was that the respective random matrix has a differentiable density. This was assumed only in order to solve a functional equation, whose solution was not known under weaker assumptions. Therefore, this assumption may be reduced to the existence of continuous densities.

An analogous assumption was imposed on the densities in the recent paper of Bobecka [2], where the multivariate MY property on trees is considered-see [2, Theorem 4.3]. Thanks to the solution of (16) under weaker assumptions, this theorem holds true if we assume continuity of densities only.

Acknowledgements This research was partially supported by NCN Grant No. 2012/05/B/ST1/00554.

Open Access This article is distributed under the terms of the Creative Commons Attribution 4.0 International License (http://creativecommons.org/licenses/by/4.0/), which permits unrestricted use, distribution, and reproduction in any medium, provided you give appropriate credit to the original author(s) and the source, provide a link to the Creative Commons license, and indicate if changes were made.

\section{References}

1. Bernadac, É.: Random continued fractions and inverse Gaussian distribution on a symmetric cone. J. Theor. Probab. 8(2), 221-259 (1995)

2. Bobecka, K.: The Matsumoto-Yor property on trees for matrix variates of different dimensions. J. Multivar. Anal. 141, 22-34 (2015)

3. Chou, C.-W., Huang, W.-J.: On characterizations of the gamma and generalized inverse Gaussian distributions. Stat. Probab. Lett. 69(4), 381-388 (2004)

4. Faraut, J., Korányi, A.: Analysis on Symmetric Cones. Oxford Mathematical Monographs. The Clarendon Press Oxford University Press, New York (1994). Oxford Science Publications

5. Herz, C.S.: Bessel functions of matrix argument. Ann. Math. 2(61), 474-523 (1955)

6. Kołodziejek, B.: The Lukacs-Olkin-Rubin theorem on symmetric cones through Gleason's theorem. Stud. Math. 217(1), 1-17 (2013)

7. Kołodziejek, B.: The Lukacs-Olkin-Rubin theorem on symmetric cones without invariance of the "Quotient". J. Theor. Probab. (2014). doi:10.1007/s10959-014-0587-3

8. Kołodziejek, B.: Multiplicative Cauchy functional equation on symmetric cones. Aequ. Math. 89(4), 1075-1094 (2015)

9. Koudou, A.E.: A Matsumoto-Yor property for Kummer and Wishart random matrices. Stat. Probab. Lett. 82(11), 1903-1907 (2012)

10. Koudou, A.E., Vallois, P.: Independence properties of the Matsumoto-Yor type. Bernoulli 18(1), 119136 (2012)

11. Kuczma, M.: An Introduction to the Theory of Functional Equations and Inequalities, 2 nd edn. Birkhäuser Verlag, Basel (2009). Cauchy's equation and Jensen's inequality, Edited and with a preface by Attila Gilányi

12. Letac, G.: Symmetric cones as Gelfand pairs: probabilistic applications. In: Probability on algebraic structures (Gainesville, FL, 1999), volume 261 of Contemp. Math., pp. 109-119. American Mathematical Society, Providence, RI (2000)

13. Letac, G., Wesołowski, J.: An independence property for the product of GIG and gamma laws. Ann. Probab. 28(3), 1371-1383 (2000)

14. Massam, H., Wesołowski, J.: The Matsumoto-Yor property and the structure of the Wishart distribution. J. Multivar. Anal. 97(1), 103-123 (2006) 
15. Matsumoto, H., Yor, M.: An analogue of Pitman's $2 M-X$ theorem for exponential Wiener functionals. II. The role of the generalized inverse Gaussian laws. Nagoya Math. J. 162, 65-86 (2001)

16. Matsumoto, H., Yor, M.: Interpretation via Brownian motion of some independence properties between GIG and gamma variables. Stat. Probab. Lett. 61(3), 253-259 (2003)

17. Wesołowski, J.: The Matsumoto-Yor independence property for GIG and gamma laws, revisited. Math. Proc. Camb. Philos. Soc. 133(1), 153-161 (2002a)

18. Wesołowski, J.: On a functional equation related to the Matsumoto-Yor property. Aequ. Math. 63(3), 245-250 (2002b) 